\title{
Green tea extract, epigallocatechin-3-gallate, inhibits the growth and invasive ability of human glioma cells
}

\author{
WEI ZHANG and JIANPING JIA

\begin{abstract}
Department of Neurology, Xuan Wu Hospital of the Capital University of Medical Sciences, 45 Changchun Street, Beijing 100053, P.R. China
\end{abstract}

Received March 17, 2008; Accepted May 23, 2008

DOI: $10.3892 / \mathrm{mmr} 00000021$

\begin{abstract}
Epigallocatechin-3-gallate (EGCG) is a major constituent of green tea, but its effects on glioma cell growth have not been reported. The aim of this study was to investigate the anticancer effect of EGCG on the growth and invasive ability of glioma cells, as well as the molecular mechanisms responsible for it. Two glioma cell lines were treated with EGCG, and its effect on cell proliferation and invasive ability was studied using the MTT, Matrigel invasion and 3-D collagen colony forming assays. Our results demonstrate that EGCG treatment leads to a decrease in cell viability and the S-phase cell fraction in a dose-dependent manner. In addition, invasive ability was significantly suppressed in the EGCGtreated cells. Furthermore, the anticancer effect of EGCG was associated with increased expression of p27 and E-cadherin. Our results suggest that EGCG is a potential anticancer agent against glioma, and that its effect may be mediated through the upregulation of p27 and E-cadherin.
\end{abstract}

\section{Introduction}

Green tea is an aqueous infusion of dried unfermented leaves derived from the Camellia sinensis plant. It is a popular beverage in Asia, especially in China and Japan, and its consumption is associated with a reduced risk of several types of cancer, including that of the stomach, esophagus, prostate and lung (1). It has been reported that the quantity of green tea consumed affects its reduction of cancer risk and the delaying of cancer outbreak and recurrence. It acts as an antioxidant, antiproliferative, antitumor and anti-angiogenic agent, and is thus a novel candidate for chemoprevention (2). In addition, many studies using both animal models and human cancer cell lines have demonstrated that green tea has chemopreventive effects on a wide range of malignancies, including prostate $(3,4)$, breast $(4)$, lung (5) and skin (6)

Correspondence to: Professor Jianping Jia, Department of Neurology, Xuan Wu Hospital of the Capital Medical University of Medical Sciences, 45 Changchun Street, Beijing 100053, P.R. China E-mail: jiaxuanwu@126.com

Key words: epigallocatechin-3-gallate, human glioma cells, growth, invasion cancers. This suggests that green tea has a wide range of anticancer activity against multiple cancer types. It contains many polyphenolic compounds, most abundant of which is the constituent known as epigallocatechin-3-gallate (EGCG), accounting for approximately $50 \%$ of the total polyphenol catechins (7). Mechanistic studies have indicated that EGCG exerts various anticancer effects, including the suppression of growth factor-mediated proliferation (8), the inhibition of transformation (9) and the repression of angiogenesis (10). In vitro studies have demonstrated that tea polyphenols, especially EGCG, cause growth inhibition and apoptosis in many types of human tumor cell lines, including melanoma, breast cancer, lung cancer, leukemia and colon cancer $(11,12)$. This suggests that EGCG may also be a chemotherapeutic agent for the suppression of the growth of many types of human cancer.

Malignant gliomas are relatively uncommon but lethal cancers. Despite recent research efforts in cancer therapy, the prognosis for patients with malignant gliomas has remained poor. A combination of radiotherapy and chemotherapy has been used as a therapeutic approach, but patient response rates are low. Recently, it has been demonstrated that a combination of EGCG with radiation in human glioma cell lines leads to increased radiosensitivity (13). The aim of this study was to investigate the effect of EGCG on the growth and invasive ability of glioma cells. Two glioma cell lines were treated with EGCG, and the effect on cell proliferation and invasive ability was studied. Additionally, the molecular mechanisms responsible for this effect were investigated.

\section{Materials and methods}

Cell lines. Two glioma cell lines, U-138MG and U-251MG (American Type Culture Collection), were cultured in RPMI1640 (Sigma) containing 5\% FCS (Life Technologies Inc.), $0.03 \mathrm{mg} / \mathrm{ml}$ penicillin and $0.05 \mathrm{mg} / \mathrm{ml}$ streptomycin (Sigma) at $37^{\circ} \mathrm{C}$ in a humidified atmosphere of $5 \% \mathrm{CO}_{2}$. Green tea epicatechin isomer (-)-EGCG with a purity of $>95 \%$ was purchased from Sigma Chemical Co. (USA).

3-(4,5-Dimethylthiazol-2-yl)-2,5-diphenyltetrazolium bromide (MTT) assay. Cells were seeded on a 96-well plate at a cell density of 2000 cells/well, incubated overnight to allow cells to attach, and then incubated with different concentrations of EGCG (up to $200 \mu \mathrm{M}$ ) for 6 days. Following this, the medium was removed and cells were incubated with $1 \mathrm{mg} / \mathrm{ml} \mathrm{MTT}$ solution for $4 \mathrm{~h}$ to allow the formation of a formazan precipi- 
A.

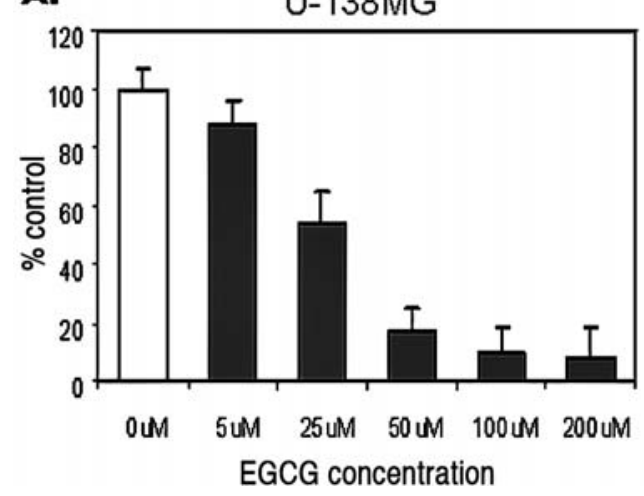

B.

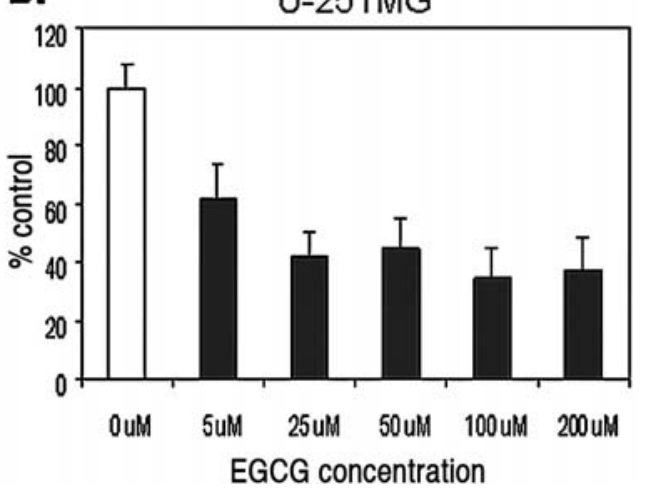

Figure 1. Effect of EGCG on cell proliferation. Cells treated for 6 days with increasing concentrations of the green tea component showed dose-dependent inhibition of cell growth. EGCG inhibited the cell growth rate. Error bars indicate the SEM.

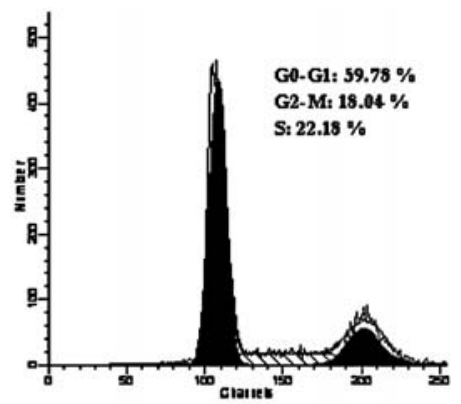

Control

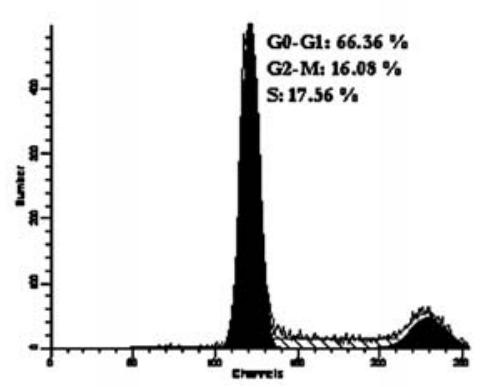

EGCG $24 \mathrm{~h}$

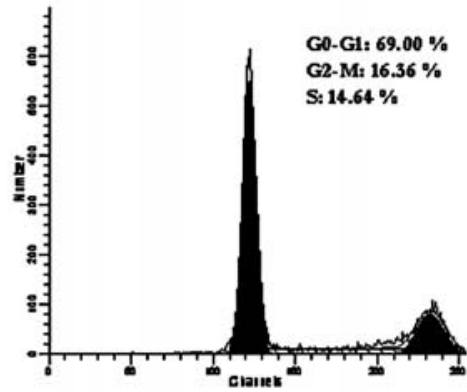

EGCG $48 \mathrm{~h}$

Figure 2. Effect of EGCG on cell cycle distribution. U-138MG cells were treated with EGCG for the indicated times, and cell cycle analysis was performed.

tate, subsequently dissolved in dimethyl sulfoxide. The absorbance in each well was read by a microplate reader at $540 \mathrm{~nm}$.

Flow cytometric analysis. Floating and adherent cells were fixed in $1 \mathrm{ml} 70 \%$ ethanol at $-20^{\circ} \mathrm{C}$ for $12 \mathrm{~h}$ and stored. Cells were later rehydrated in $1 \mathrm{ml} \mathrm{PBS} \mathrm{pH} 7.4$ and stored at $4^{\circ} \mathrm{C}$ overnight. Prior to analysis, cells were treated with $0.5 \mu \mathrm{g} / \mathrm{ml}$ RNaseA (Sigma) and stained with propidium iodide $(1 \mu \mathrm{g} / \mathrm{ml})$ for $30 \mathrm{~min}$ at $37^{\circ} \mathrm{C}$. Cell cycle analysis was conducted using the flow cytometer EPICS profile analyzer (Coulter, Miami, FL) and Modfit LT2.0 software (Verity Software House, Topsham, ME). Each experiment was repeated at least four times.

Matrigel invasion assay. To prepare an in vitro basement membrane, commercial cell culture invasion inserts $(8-\mu \mathrm{m}$ pore size; Millipore) were coated with Matrigel (120 $\mu \mathrm{g} / \mathrm{insert}$; Becton Dickinson) for $24 \mathrm{~h}$. The assay was activated by the addition of 20,000 cells in $200 \mu 1$ of $0.1 \%$ BSA-RPMI in the upper chamber with or without EGCG $(100 \mu \mathrm{M}), 400 \mu \mathrm{l}$ fibronectin $(10 \mu \mathrm{g} / \mathrm{ml})$ and $400 \mu 15 \%$ FCS RPMI in the lower chamber as the chemoattractant to induce invasion. Cells were incubated for $24 \mathrm{~h}$ at $37^{\circ} \mathrm{C}$ and $5 \% \mathrm{CO}_{2}$, and insert membranes were prepared for microscopic samples. Membranes were fixed for 10 min with $10 \%$ formaldehyde (J.T. Backer) and then stained with Mayer's hematoxylin (Zymed) and 1\% w/v aqueous eosin solution. The cells on the upper surface of the membrane were wiped off with cotton wool. After washing, the membranes were cut from the inserts and mounted with Depex. The number of cells on the lower surface of the membrane was counted using a PC-based image-analyzing system (Stereo Investigator) attached to a Nikon microscope with a x12.5 objective and x10 eyepieces. Representative areas of the invasive cells were photographed with a cooled color CCD camera (Optronics) and digitally stored on a desktop computer. Experiments were repeated three times, and each treatment was carried out in quadruplicate.

3-Dimension collagen colony forming assay. The cell suspension $\left(200 \mu \mathrm{l} ; 3 \times 10^{4}\right.$ cells $\left./ \mathrm{ml}\right)$ was mixed with $200 \mu \mathrm{l}$ of cold rat tail collagen type I ( $3.60 \mathrm{mg} / \mathrm{ml}$; BD Biosciences, MA). The mixed cell solution in collagen was plated as droplets in a $60-\mathrm{mm}$ Petri-dish and air dried at room temperature. RPMI1640 medium with 3\% FCS with or without EGCG was added to each dish containing the semi-solid collagen-cell droplets. EGCG-treated and control samples were incubated at $37^{\circ} \mathrm{C}$ for 3-5 days. Cell morphology was observed using a phase contrast microscope, and images were captured under x200 magnification. A total of 500 colonies were counted in each experiment, and the percentage of colonies with elongated morphology was calculated. Each experiment was repeated at least three times. Error bars in figures indicate the standard deviation.

Western blot analysis. Protein was extracted with a modified radioimmunoprecipitation (RIPA) buffer (50 mM Tris- $\mathrm{HCl}$; 

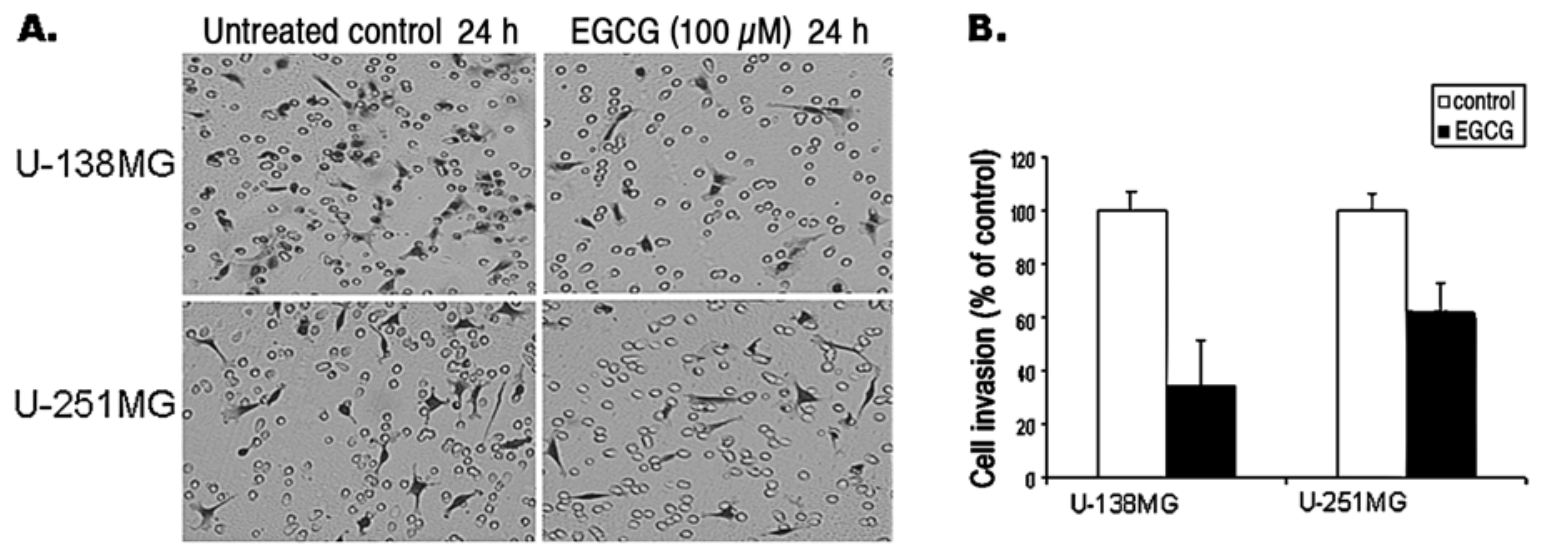

Figure 3. Effect of EGCG on cell invasion. The cells $\left(2 \times 10^{4}\right)$ were seeded on a Matrigel-precoated filter in an invasion chamber with or without EGCG (100 $\left.\mu \mathrm{M}\right)$. After a 24-h incubation, the cells were fixed and stained. Cells from the upper side of the filter were removed, and cells from the lower surface of the filter were counted using a microscope. Magnification, x125. EGCG inhibited the invasive ability of the cells. (A) Representative images of the Matrigel assay results. (B) Summary of cell invasive ability (\%).

A.

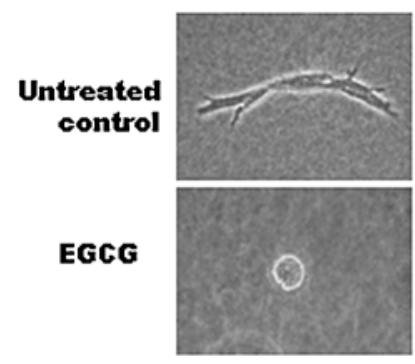

B.

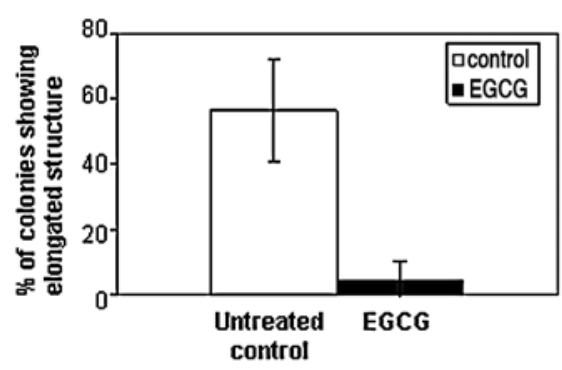

U-251MG

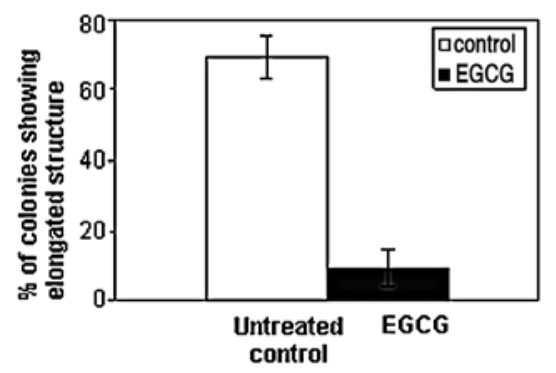

Figure 4. Effect of EGCG on 3-D gel invasive ability. EGCG treatment led to the suppression of the ability of cells to grow in semi-solid medium, indicating a decreased ability to invade neighboring tissues.

pH 7.4), $1 \%$ NP-40, $1 \%$ Na-deoxycholate, $150 \mathrm{mM} \mathrm{NaCl}$, $1 \mathrm{mM}$ EDTA, $0.1 \%$ SDS, $1 \mathrm{mM} \mathrm{Na} \mathrm{VO}_{4}$ and $1 \mathrm{mM} \mathrm{NaF}$ ) containing proteinase inhibitors (Complete Protease Inhibitor Cocktail Tablets, Roche). Protein concentrations were measured using the DC protein assay kit (Bio-Rad Laboratories Inc.). Proteins $(20 \mu \mathrm{g})$ were separated by electrophoresis on a $12.5 \%$ SDS-polyacrylamide gel (SDS-PAGE) and blotted onto nitrocellulose membrane (Amersham Piscataway, NJ, USA). After blocking with $10 \%$ non-fat dry milk, the blots were respectively incubated with primary antibodies against p27, p53, p21 and E-cadherin (1:1000; BD Biosciences), and then incubated with horseradish peroxidase (HRP)-conjugated secondary antibody (Amersham). Signals were detected using ECL Plus Western blot detection reagents (Amersham).

Statistical analysis. The collected data were analyzed using one-way ANOVA followed by the Newman-Keuls multiple comparison test. Statistical analysis was performed using Prism version 3.0 statistical software (GraphPad Software, San Diego, CA). A p-value $<0.05$ was considered significant.

\section{Results}

Effect of EGCG on proliferation of glioma cells. Two glioma cell lines, U-138MG and U-251MG, were treated with 5 doses of EGCG, and the effect on cell growth rate was measured by
MTT. As shown in Fig. 1, EGCG treatment led to decreased cell viability in a dose-dependent manner. U-138MG cells were also found to be slightly more sensitive to EGCGinduced growth suppression than U-251MG ones. To further confirm these results, we performed cell cycle analysis. As shown in Fig. 2, the $\mathrm{S}$ phase fraction of $\mathrm{U}-138 \mathrm{MG}$ cells was decreased from $22 \%$ at the 0 time-point to $14 \%$ after $48 \mathrm{~h}$ of EGCG treatment $(25 \mu \mathrm{M})$. This decreased $\mathrm{S}$ phase cell fraction was correlated with an increase in G1 phase cells (from 59 to $69 \%$ ). The results suggest that EGCG is able to suppress the growth of glioma cells in a dose- and time-dependent manner.

Effect of EGCG on the invasive ability of glioma cells. We investigated whether EGCG had any effect on the invasive ability of the U-138MG and U-251MG glioma cell lines. Matrigel invasion assay revealed that EGCG treatment led to the decreased ability of the cells to penetrate the cell culture filter membrane, suggesting that EGCG suppressed the invasion of glioma cells (Fig. 3A). Further analysis (Fig. 3B) showed that there was a significant decrease in the invasive ability of the EGCG-treated cells compared to the untreated control cells $(\mathrm{p}<0.05)$ in both lines, suggesting that EGCG suppressed the metastatic ability of these cells. To further confirm these results, we performed a 3-D collagen invasion assay. As shown in Fig. 4A, while the untreated cells grew inside the semi-solid collagen, a tightly packed colony was 


\section{$\mathrm{U}-138 \mathrm{MG}$}

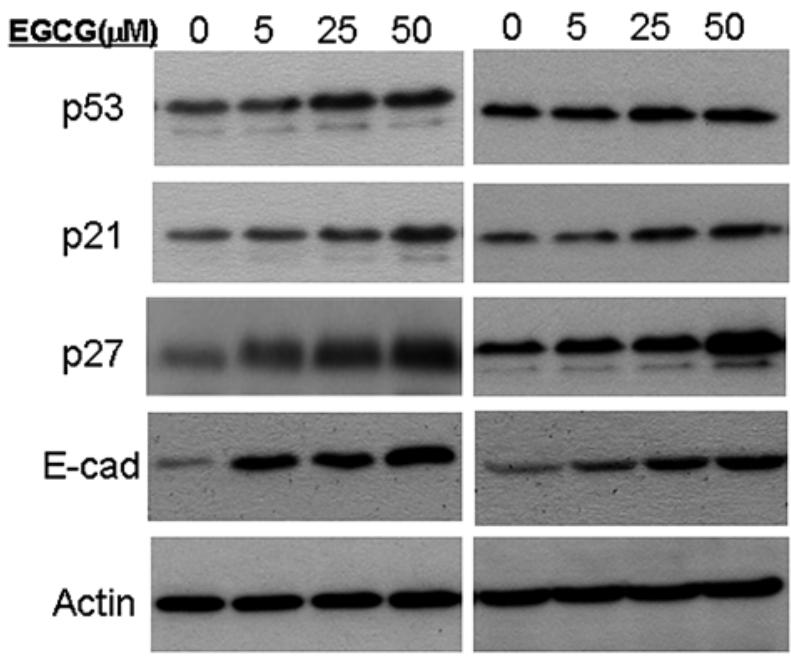

Figure 5. Western blot analysis showing the effect of EGCG on p53, p21, p27 and E-cadherin (E-cad) in U-138MG and U-251MG cells. Cells were treated with EGCG for $48 \mathrm{~h}$, and cell lysates were isolated. Western blotting was performed as described in Materials and methods.

observed in the cells treated with EGCG. This demonstrates that EGCG inhibited the ability of the cells to invade the semi-solid medium and indicates that it was able to suppress glioma cell invasion of other tissues.

Effect of EGCG on the expression of p53, p21, p27 and Ecadherin. To further study the molecular mechanisms responsible for effect of EGCG on cell growth and invasion, we performed Western blotting. To determine whether p53, p21 and p27 played a role in EGCG-induced growth arrest, we treated both cell lines with 3 doses of EGCG for $24 \mathrm{~h}$. In the $\mathrm{U}-138 \mathrm{MG}$ cells, p53 was upregulated in a dose-dependent manner, but the same effect was not observed in the $\mathrm{U}-251 \mathrm{MG}$ cells (Fig. 5). In addition, the level of p21 was upregulated in the U-138MG cell line, correlated with the upregulation of p53. These results suggest that EGCGinduced growth arrest is dependent on the activation of p53. However, due to a 553 mutation in U-251MG cells as previously reported (14), an anticipated slight alteration in p53 levels in these cells was observed. In addition, we found that p27 was increased in a dose-dependent manner in both cell lines, suggesting that EGCG-induced cell growth arrest is associated with the upregulation of p27. E-cadherin is one of the cell surface adhesion molecules that plays a key role in preventing the invasion of cancer cells (15). We found that there was an increase in E-cadherin expression after treatment with EGCG in both cell lines.

\section{Discussion}

In the present study, we demonstrated that EGCG treatment alone was able to suppress the growth of glioma cells. In addition, this growth inhibitory effect was mediated through both p53-dependent and -independent pathways. In the cells with wild-type p53, such as U-138MG, the EGCG-induced inhibitory effect was mediated through the activation of p53, which in turn led to the induction of p21 and subsequent cell cycle G1 phase arrest. In the cells with a p53 mutation, such as the U-251MG cells, the EGCG-induced growth suppression may have been mediated through p53-independent upregulation of $\mathrm{p} 27$, a CDK inhibitor which plays a key role in the induction of cell cycle $\mathrm{G} 1$ phase arrest. Our results provide evidence to suggest that EGCG may act as an agent against the growth of glioma cells. It has been reported that EGCG is able to induce radiosensitivity in glioma cells (13). Our results in this study suggest that EGCG could be used as a single agent to achieve an anticancer effect. The anticancer effect of EGCG has been reported in several types of cancers (16-18). In this study, we provided evidence to suggest that its action is not dependent on a functional p53 in glioma cells. This observation is very significant, as a p53 mutation is a common event in glioma cells, particularly in advanced cancers. Therefore, it is possible that EGCG may be a potential effective agent for the treatment of advanced cancers.

Metastasis is one of the main causes of cancer-related death, and the suppression of cancer invasion is consistently a main target for the development of new anticancer drugs. In this study, we found that EGCG treatment reduced invasive ability and restored E-cadherin expression in both our cell lines. Since E-cadherin downregulation is one of the commonly observed characteristics in metastatic cancers, it is possible that the anti-metastatic effect of EGCG is regulated through the upregulation of E-cadherin. It has been reported that EGCG is able to upregulate E-cadherin in an animal model of intestinal cancer (19). The present study was the first to demonstrate this effect in human glioma cells, though the mechanism behind this upregulation has yet to be elucidated. EGCG has also been reported to reactivate E-cadherin gene expression through the removal of methylation in the promoter region of the E-cadherin gene. Since upregulation of E-cadherin has been suggested as a therapeutic target for the treatment of metastatic cancer, our results suggest that EGCG has potential as an anticancer drug capable of inhibiting the invasive ability of glioma cells.

In summary, we established the potential anticancer effect of EGCG on two glioma cancer cell lines, and demonstrated its effect on the suppression of cancer cell growth and invasive ability. This may be mediated through the induction of cell cycle G1 phase arrest and the upregulation of E-cadherin. Our results suggest the novel therapeutic potential of EGCG for the treatment of advanced glioma.

\section{References}

1. Park OJ and Surh YJ: Chemopreventive potential of epigallocatechin gallate and genistein: evidence from epidemiological and laboratory studies. Toxicol Lett 150: 43-56, 2004.

2. Lambert JD and Yang CS: Mechanisms of cancer prevention by tea constituents. J Nutr 133: S3262-S3267, 2003.

3. Caporali A, Davalli P, Astancolle S, D'Arca D, Brausi M, Bettuzzi S and Corti A: The chemopreventive action of catechins in the TRAMP mouse model of prostate carcinogenesis is accompanied by clusterin over-expression. Carcinogenesis 25 : 2217-2224, 2004

4. Stuart EC, Scandlyn MJ and Rosengren RJ: Role of epigallocatechin gallate (EGCG) in the treatment of breast and prostate cancer. Life Sci 79: 2329-2336, 2006.

5. Chung FL: The prevention of lung cancer induced by a tobaccospecific carcinogen in rodents by green and black tea. Proc Soc Exp Biol Med 220: 244-248, 1999. 
6. Katiyar SK, Matsui MS, Elmets CA and Mukhtar H: Polyphenolic antioxidant (-)-epigallocatechin-3-gallate from green tea reduces UVB-induced inflammatory responses and infiltration of leukocytes in human skin. Photochem Photobiol 69: 148-153, 1999.

7. Khan N, Afaq F, Saleem M, Ahmad N and Mukhtar H: Targeting multiple signaling pathways by green tea polyphenol (-)-epigallocatechin-3-gallate. Cancer Res 66: 2500-2505, 2006.

8. Ermakova S, Choi BY, Choi HS, Kang BS, Bode AM and Dong Z: The intermediate filament protein vimentin is a new target for epigallocatechin gallate. J Biol Chem 280: 16882-16890, 2005.

9. Sachinidis A, Seul C, Seewald S, Ko Y and Vetter H: Green tea compounds inhibit tyrosine phosphorylation of PDGF betareceptor and transformation of A172 human glioblastoma. FEBS Lett 471: 51-55, 2000.

10. Jung YD and Ellis LM: Inhibition of tumour invasion and angiogenesis by epigallocatechin gallate (EGCG), a major component of green tea. Int J Exp Pathol 82: 309-316, 2001.

11. Lyn-Cook BD, Rogers T, Yan Y, Blann EB, Kadlubar FF and Hammons GJ: Chemopreventive effects of tea extracts and various components on human pancreatic and prostate tumor cells in vitro. Nutr Cancer 35: 80-86, 1999.

12. Yang CS, Lambert JD, Hou Z, Ju J, Lu G and Hao X: Molecular targets for the cancer preventive activity of tea polyphenols. Mol Carcinog 45: 431-435, 2006.
13. McLaughlin N, Annabi B, Bouzeghrane M, Temme A, Bahary JP, Moumdjian R and Béliveau R: The survivin-mediated radioresistant phenotype of glioblastomas is regulated by RhoA and inhibited by the green tea polyphenol (-)-epigallocatechin-3gallate. Brain Res 1071: 1-9, 2006.

14. Lang FF, Yung WK, Raju U, Libunao F, Terry NH and Tofilon PJ: Enhancement of radiosensitivity of wild-type p53 human glioma cells by adenovirus-mediated delivery of the $\mathrm{p} 53$ gene. J Neurosurg 89: 125-132, 1998.

15. Nam JS, Hirohashi S and Wakefield LM: Dysadherin: A new player in cancer progression. Cancer Lett 255: 161-169, 2007.

16. Sadava D, Whitlock E and Kane SE: The green tea polyphenol, epigallocatechin-3-gallate inhibits telomerase and induces apoptosis in drug-resistant lung cancer cells. Biochem Biophys Res Commun 360: 233-237, 2007.

17. Belguise K, Guo S and Sonenshein GE: Activation of FOXO3a by the green tea polyphenol epigallocatechin-3-gallate induces estrogen receptor \{alpha\} expression reversing invasive phenotype of breast cancer cells. Cancer Res 67: 5763-5770, 2007.

18. Shankar S, Ganapathy S and Srivastava RK: Green tea polyphenols: biology and therapeutic implications in cancer. Front Biosci 12: 4881-4899, 2007.

19. Ju J, Hong J, Zhou JN, Pan Z, Bose M, Liao J, Yang GY, Liu YY, Hou Z, Lin Y, Ma J, Shih WJ, Carothers AM and Yang CS: Inhibition of intestinal tumorigenesis in Apcmin/+ mice by (-)epigallocatechin-3-gallate, the major catechin in green tea. Cancer Res 65: 10623-10631, 2005. 